\title{
Front Matter: Volume 6930
}

, "Front Matter: Volume 6930," Proc. SPIE 6930, Industrial and Commercial Applications of Smart Structures Technologies 2008, 693001 (12 May 2008); doi: $10.1117 / 12.800811$ Health Monitoring, 2008, San Diego, California, United States 


\title{
PROCEEDINGS OF SPIE
}

\section{Industrial and Commercial Applications of Smart Structures Technologies 2008}

\author{
L. Porter Davis \\ Benjamin Kyle Henderson \\ M. Brett McMickell \\ Editors
}

10-11 March 2008

San Diego, California, USA

Sponsored by

SPIE

Cosponsored by

American Society of Mechanical Engineers (USA)

Cooperating Organizations

Intelligent Materials Forum (Japan)

Jet Propulsion Laboratory (USA)

National Science Foundation (USA)

Published by

SPIE 
The papers included in this volume were part of the technical conference cited on the cover and title page. Papers were selected and subject to review by the editors and conference program committee. Some conference presentations may not be available for publication. The papers published in these proceedings reflect the work and thoughts of the authors and are published herein as submitted. The publisher is not responsible for the validity of the information or for any outcomes resulting from reliance thereon.

Please use the following format to cite material from this book:

Author(s), "Title of Paper," in Industrial and Commercial Applications of Smart Structures Technologies 2008, edited by L. Porter Davis, Benjamin Kyle Henderson, M. Brett McMickell, Proceedings of SPIE Vol. 6930 (SPIE, Bellingham, WA, 2008) Article CID Number.

ISSN 0277-786X

ISBN 9780819471161

Published by

SPIE

P.O. Box 10, Bellingham, Washington $98227-0010$ USA

Telephone +1 3606763290 (Pacific Time) · Fax +1 3606471445

SPIE.org

Copyright (c) 2008, Society of Photo-Optical Instrumentation Engineers

Copying of material in this book for internal or personal use, or for the internal or personal use of specific clients, beyond the fair use provisions granted by the U.S. Copyright Law is authorized by SPIE subject to payment of copying fees. The Transactional Reporting Service base fee for this volume is $\$ 18.00$ per article (or portion thereof), which should be paid directly to the Copyright Clearance Center (CCC), 222 Rosewood Drive, Danvers, MA 01923. Payment may also be made electronically through CCC Online at copyright.com. Other copying for republication, resale, advertising or promotion, or any form of systematic or multiple reproduction of any material in this book is prohibited except with permission in writing from the publisher. The CCC fee code is 0277-786X/08/\$18.00.

Printed in the United States of America.

Publication of record for individual papers is online in the SPIE Digital Library.

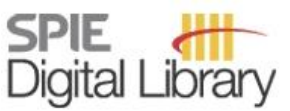

SPIEDigitalLibrary.org

Paper Numbering: Proceedings of SPIE follow an e-First publication model, with papers published first online and then in print and on CD-ROM. Papers are published as they are submitted and meet publication criteria. A unique, consistent, permanent citation identifier (CID) number is assigned to each article at the time of the first publication. Utilization of CIDs allows articles to be fully citable as soon they are published online, and connects the same identifier to all online, print, and electronic versions of the publication. SPIE uses a six-digit CID article numbering system in which:

- The first four digits correspond to the SPIE volume number.

- The last two digits indicate publication order within the volume using a Base 36 numbering system employing both numerals and letters. These two-number sets start with $00,01,02,03,04,05$, 06, 07, 08, 09, OA, OB ... 0Z, followed by 10-1Z, 20-2Z, etc.

The CID number appears on each page of the manuscript. The complete citation is used on the first page, and an abbreviated version on subsequent pages. Numbers in the index correspond to the last two digits of the six-digit CID number. 


\section{Contents}

vii Symposium Committee

ix Conference Committee

\section{SESSION 1 AUTOMOTIVE APPLICATIONS I}

693003 Active material reversible attachments: shape memory polymer based [6930-02] T. Stanford, W. Barvosa-Carter, HRL Labs., LLC (USA); A. L. Browne, N. L. Johnson, General Motors R\&D (USA)

693004 Behavioral model and experimental validation for a spool-packaged shape memory alloy actuator [6930-03]

J. A. Redmond, D. Brei, J. Luntz, Univ. of Michigan (USA); A. L. Browne, N. L. Johnson, General Motors R\&D Ctr. (USA)

693005 Shape memory alloy resetable spring lift for pedestrian protection [6930-04]

B. M. Barnes, D. E. Brei, J. E. Luntz, Univ. of Michigan (USA); K. Strom, A. L. Browne,

N. Johnson, General Motors R\&D (USA)

693006 Experimental investigation of active adaptability of the SMArt (SMA reseTtable) dualchamber pneumatic lift device for pedestrian protection [6930-05]

J. Otten, J. Luntz, D. Brei, Univ. of Michigan (USA); K. A. Strom, A. L. Browne, N. L. Johnson, General Motors R\&D (USA)

\section{SESSION 2 AUTOMOTIVE APPLICATIONS II}

6930 0A Nonlinear force feedback control of piezoelectric-hydraulic pump actuator for automotive transmission shift control [6930-09]

G.-W. Kim, K. W. Wang, The Pennsylvania State Univ. (USA)

6930 OB Motorcycle waste heat energy harvesting [6930-10]

A. D. Schlichting, S. R. Anton, D. J. Inman, Virginia Polytechnic Institute and State Univ. (USA)

6930 OC Thermoelectrics as elements of hybrid-electric vehicle thermal energy systems [6930-11] L. Headings, G. Washington, C. M. Jaworski, The Ohio State Univ. (USA)

\section{SESSION 3 VIBRATION DAMPING APPLICATIONS}

6930 OE A practical design method for TEP-GT MRFD [6930-13]

J. Li, Dalian Univ. of Technology (China); X. Guan, Harbin Institute of Technology (China);

J. Ou, Dalian Univ. of Technology (China) and Harbin Institute of Technology (China) 
6930 OF Active component and control design for torsional mode vibration reduction for a parallel kinematic machine tool structure [6930-15]

R. Neugebauer, V. Wittstock, A. Bucht, A. Illgen, Fraunhofer-Institute for Machine Tools and Forming Technology (Germany)

6930 OG Manufacturing technique for robust and modular smart composites [6930-16]

G. Kessenich, B. Pletner, IPTRADE, Inc. (USA)

\section{SESSION 4 SPACE APPLICATIONS}

$6930 \mathrm{OH}$ Thermo-mechanical analysis of thin membranes and application in active flatness control design [6930-17]

X. Wang, C. Sulik, W. Zheng, Y.-R. Hu, Canadian Space Agency (Canada)

69300 ol Development of a satellite structural architecture for operationally responsive space [6930-18]

B. J. Arritt, S. J. Buckley, J. M. Ganley, J. S. Welsh, B. K. Henderson, M. E. Lyall, A. D. Williams, Air Force Research Lab. (USA); J. C. Preble, J. DiPalma, SpaceWorks, Inc. (USA); G. Mehle, CSA Engineering, Inc. (USA); R. Roopnarine, Honeybee Robotics Spacecraft Mechanisms Corp. (USA)

\section{SESSION 5 AEROSPACE APPLICATIONS}

6930 OK Design of a piezoceramic-driven synthetic-jet actuator for aerodynamic performance improvement [6930-20]

R. Rusovici, Florida Institute of Technology (USA); C. Offord, The Boeing Co. (USA);

R. Honour, F. Goto, P. Louderback, C. Phelps, Florida Institute of Technology (USA)

6930 OL Development of a 1/4-scale NiTinol actuator for reconfigurable structures [6930-21] D. J. Arbogast, R. T. Ruggeri, Boeing Phantom Works (USA); R. C. Bussom, The Boeing Co. (USA)

6930 OM Development of highly reliable advanced grid structure (HRAGS) demonstrator using FBG sensors [6930-22]

H. Takeya, K. Sekine, M. Kume, T. Ozaki, Mitsubishi Electric Corp. (Japan); N. Takeda, The Univ. of Tokyo (Japan); N. Tajima, R\&D Institute of Metals and Composites for Future Industries (Japan)

6930 ON Stability in hovering ornithopter flight [6930-23]

J. M. Dietl, E. Garcia, Cornell Univ. (USA)

693000 An experimental and analytical study of a flow vectoring airfoil via macro-fiber-composite actuators [6930-24]

O. Bilgen, K. B. Kochersberger, D. J. Inman, Virginia Polytechnic Institute and State Univ. (USA) 
6930 OP Development and test of an HTSMA supersonic inlet ramp actuator [6930-25]

T. R. Quackenbush, Continuum Dynamics, Inc. (USA); B. F. Carpenter, BBC Consultants

(USA); A. H. Boschitsch, P. V. Danilov, Continuum Dynamics, Inc. (USA)

$69300 Q$ Stabilizing shape memory alloy actuator performance through cyclic shakedown: an empirical study [6930-26]

H. Sun, A. Pathak, J. Luntz, D. Brei, Univ. of Michigan (USA); P. W. Alexander, N. L. Johnson, General Motors R\&D (USA)

$6930 \mathrm{OR}$ High temperature shape memory alloy actuators through mechanical treatments for an oil \& gas down-hole valve [6930-27]

J. Gore, A. Bowles, M. Maylin, L. Chandrasekaran, QinetiQ Ltd. (United Kingdom);

D. Forsyth, M. Buyers, Omega Completion Technology Ltd. (United Kingdom)

6930 OS Spray forming of NiTi and NiTiPd shape-memory alloys [6930-28]

R. Smith, Materials Resources International (USA); J. Mabe, R. Ruggeri, Boeing Phantom Works (USA); R. Noebe, NASA Glenn Research Ctr. (USA)

6930 OT Variable area jet nozzle using shape memory alloy actuators in an antagonistic design [6930-29]

J. H. Mabe, Boeing Phantom Works (USA); F. T. Calkins, M. B. Alkislar, Boeing Commercial Airplane Co. (USA)

\section{SESSION 7 MEDICAL AND OPTICAL APPLICATIONS}

6930 OU New configurations of oscillatory flow pumps using bimorph piezoelectric actuators [6930-30]

S. L. Vatanabe, Escola Politécnica da Univ. de São Paulo (Brazill); R. F. Pires, Inoveo Indústria, Comérico e Serviços de Automação de Sistemas Ltda. (Brazil); P. H. Nakasone, E. C. N. Silva, Escola Politécnica da Univ. de São Paulo (Brazil)

$6930 \mathrm{OV}$ Study of oscillatory piezoelectric flow pumps using bimorph actuators with different tip geometries [6930-31]

R. F. Pires, Inoveo Indústria, Comércio e Serviços de Automação de Sistemas Ltda. (Brazil); A. A. Koga, P. H. Nakasone, E. C. N. Silva, Escola Politécnica da Univ. de São Paulo (Brazil)

6930 OW Experimental investigation of waveguide dynamics for the medical invasion treatment [6930-32]

A. Bubulis, A. Palevicius, V. Jurenas, R. Bansevicius, A. Rinkevicius, Kaunas Univ. of Technology (Lithuania)

6930 oY A piezoelectric deformable mirror for intra-cavity laser adaptive optics [6930-35] C. S. Long, P. W. Loveday, CSIR Material Science and Manufacturing (South Africa); A. Forbes, CSIR National Laser Ctr. (South Africa) and Univ. of KwaZulu-Natal (South Africa)

$69300 Z$ High-power piezoelectric acoustic-electric power feedthru for metal walls [6930-36] X. Bao, W. Biederman, S. Sherrit, M. Badescu, Y. Bar-Cohen, C. Jones, J. Aldrich, Z. Chang, Jet Propulsion Lab. (USA) 
693011 Design, development, and testing of a transonic missile fin employing PBP/DEAS actuators [6930-37]

R. Barrett, R. Vos, The Univ. of Kansas (USA)

693012 The application of thermally induced multistable composites to morphing aircraft structures [6930-38]

F. Mattioni, P. M. Weaver, K. D. Potter, M. I. Friswell, Bristol Univ. (United Kingdom)

693013 A comparative study of ultrasonic micro-motors based on single crystal PMN-PT and polycrystalline PZT ceramics [6930-39]

S. A. Wilson, P. J. Rayner, Cranfield Univ. (United Kingdom); J. Gore, A. R. Bowles,

R. C. McBride, QinetiQ Ltd. (United Kingdom)

693014 Design and evaluation of bimorph and sandwich tunable frequency power harvesting devices [6930-40]

Y.-Y. Chen, National Taiwan Univ. (Taiwan); C.-S. Lai, Y.-S. Chou, C.-K. Hsu, Industrial Technology Research Institute (Taiwan); W.-J. Wu, National Taiwan Univ. (Taiwan)

693016 Adaptive structures for manipulation in clean room [6930-43]

C. Lira, F. Scarpa, Univ. of Bristol (United Kingdom)

Author Index 


\title{
Symposium Committee
}

\author{
Symposium Chairs
}

Alison B. Flatau, University of Maryland, College Park (USA)

George Y. Baaklini, NASA Glenn Research Center (USA)

Donald J. Leo, Virginia Polytechnic Institute and State University (USA)

Kara J. Peters, North Carolina State University (USA)

Executive Committee

Alison B. Flatau, University of Maryland, College Park (USA)

George Y. Baaklini, NASA Glenn Research Center (USA)

Donald J. Leo, Virginia Polytechnic Institute and State University (USA)

Kara J. Peters, North Carolina State University (USA)

Mehdi Ahmadian, Virginia Polytechnic Institute and State University (USA)

Yoseph Bar-Cohen, Jet Propulsion Laboratory (USA)

Emilio P. Calius, Industrial Research Ltd. (New Zealand)

Marcelo J. Dapino, The Ohio State University (USA)

L. Porter Davis, Honeywell, Inc. (USA)

Michael A. Demetriou, Worcester Polytechnic Institute (USA)

Aaron A. Diaz, Pacific Northwest National Laboratory (USA)

Wolfgang Ecke, IPHT Jena (Germany)

Mehrdad N. Ghasemi-Nejhad, University of Hawai'i at Manoa (USA)

Victor Giurgiutiu, University of South Carolina (USA)

B. Kyle Henderson, Air Force Research Laboratory (USA)

Kumar V. Jata, Air Force Research Laboratory (USA)

Tribikram Kundu, The University of Arizona (USA)

Douglas K. Lindner, Virginia Polytechnic Institute and State University (USA)

Ajit K. Mal, University of California, Los Angeles (USA)

M. Brett McMickell, Honeywell, Inc. (USA)

Norbert G. Meyendorf, Fraunhofer-Institut für Zerstörungsfreie

Prüfverfahren (Germany) and University of Dayton (USA)

Zoubeida Ounaies, Texas A\&M University (USA)

Andrei M. Shkel, University of California, Irvine (USA)

Peter J. Shull, The Pennsylvania State University (USA)

Masayoshi Tomizuka, University of California, Berkeley (USA)

Vijay K. Varadan, University of Arkansas (USA)

Dietmar W. Vogel, Fraunhofer-Institut für Zuverlässigkeit und Mikrointegration (Germany)

H. Felix Wu, National Institute of Standards and Technology (USA)

Chung-Bang Yun, Korea Advanced Institute of Science and Technology

(South Korea) 
Downloaded From: https://www.spiedigitallibrary.org/conference-proceedings-of-spie on 25 Apr 2023

Terms of Use: https://www.spiedigitallibrary.org/terms-of-use 


\title{
Conference Committee
}

\author{
Conference Chair
}

L. Porter Davis, Honeywell, Inc. (USA)

Conference Cochairs

Benjamin Kyle Henderson, Air Force Research Laboratory (USA)

M. Brett McMickell, Honeywell International, Inc. (USA)

Program Committee

Eric H. Anderson, CSA Engineering, Inc. (USA)

Emil V. Ardelean, Schafer Corporation (USA)

Christian Boller, The University of Sheffield (United Kingdom)

Peter C. Chen, Catholic University of America (USA)

Kevin M. Farinholt, Virginia Polytechnic Institute and State University (USA)

Xiaoyan Gong, Medical Implant Mechanics, LLC (USA)

Steven F. Griffin, Boeing-SVS, Inc. (USA)

Holger Hanselka, Fraunhofer-Institut für Betriebsfestigkeit und Systemzuverlässigkeit (Germany)

David E. Havens, Cornerstone Research Group, Inc. (USA)

Mark R. Jolly, Lord Corporation (USA)

Chad H. Joshi, Energen, Inc. (USA)

Jayanth N. Kudva, NextGen Aeronautics, Inc. (USA)

Amrita Kumar, Acellent Technologies, Inc. (USA)

Ou Ma, New Mexico State University (USA)

Anna-Maria R. McGowan, NASA Langley Research Center (USA)

Geoffrey P. McKnight, HRL Laboratories, LLC (USA)

Marc E. Regelbrugge, Rhombus Consultants Group (USA)

W. Lance Richards, NASA Dryden Flight Research Center (USA)

Brian P. Sanders, Air Force Research Laboratory (USA)

Janet M. Sater, Institute for Defense Analyses (USA)

\section{Session Chairs}

1 Automotive Applications I

Marc E. Regelbrugge, Rhombus Consultants Group (USA)

Benjamin Kyle Henderson, Air Force Research Laboratory (USA) 
2 Automotive Applications II

Janet $\mathbf{M}$. Sater, Institute for Defense Analyses (USA)

M. Brett McMickell, Honeywell International, Inc. (USA)

$3 \quad$ Vibration Damping Applications

L. Porter Davis, Honeywell, Inc. (USA)

Geoffrey P. McKnight, HRL Laboratories, LLC (USA)

$4 \quad$ Space Applications

Ou Ma, New Mexico State University (USA)

M. Brett McMickell, Honeywell International, Inc. (USA)

$5 \quad$ Aerospace Applications

W. Lance Richards, NASA Dryden Flight Research Center (USA)

M. Brett McMickell, Honeywell International, Inc. (USA)

6 Future of SMA

David E. Havens, Cornerstone Research Group, Inc. (USA)

L. Porter Davis, Honeywell, Inc. (USA)

$7 \quad$ Medical and Optical Applications

Steven F. Griffin, Boeing-SVS, Inc. (USA)

Kevin M. Farinholt, Los Alamos National Laboratory (USA) 